\title{
Deep neuromuscular blockade improves surgical conditions during low-pressure pneumoperitoneum laparoscopic donor nephrectomy
}

\author{
D. M. D. Özdemir-van Brunschot ${ }^{1}$ - A. E. Braat ${ }^{2}$ - M. F. P. van der Jagt ${ }^{1}$ • \\ G. J. Scheffer ${ }^{3}$ - C. H. Martini ${ }^{4} \cdot$ J. F. Langenhuijsen ${ }^{5}$ - R. E. Dam ${ }^{2}$. \\ V. A. Huurman' ${ }^{2}$ D. Lam $^{2}$ - F. C. d'Ancona ${ }^{5}$ A. Dahan ${ }^{4}$ M. C. Warlé1
}

Received: 7 March 2017/ Accepted: 8 June 2017/Published online: 22 June 2017

(c) The Author(s) 2017. This article is an open access publication

\begin{abstract}
Background Evidence indicates that low-pressure pneumoperitoneum (PNP) reduces postoperative pain and analgesic consumption. A lower insufflation pressure may hamper visibility and working space. The aim of the study is to investigate whether deep neuromuscular blockade (NMB) improves surgical conditions during low-pressure PNP.

Methods This study was a blinded randomized controlled multicenter trial. 34 kidney donors scheduled for laparoscopic donor nephrectomy randomly received low-pressure PNP (6 mmHg) with either deep (PTC 1-5) or moderate NMB (TOF 0-1). In case of insufficient surgical conditions, the insufflation pressure was increased stepwise. Surgical conditions were rated by the Leiden-Surgical Rating Scale (L-SRS) ranging from 1 (extremely poor) to 5 (optimal).

Results Mean surgical conditions were significantly better for patients allocated to a deep NMB (SRS 4.5 versus 4.0;
\end{abstract}

M. C. Warlé

Michiel.Warle@radboudumc.nl

1 Division of Vascular and Transplant Surgery, Department of Surgery, Radboud University Medical Centre, Geert Grooteplein-Zuid 10, 6525 GA Nijmegen, The Netherlands

2 Department of Surgery, Leiden University Medical Center, Albinusdreef 2, 2300 RC Leiden, The Netherlands

3 Department of Anesthesiology, Radboud University Medical Centre, Geert Grooteplein-Zuid10, 6525 GA Nijmegen, The Netherlands

4 Department of Anesthesiology, Leiden University Medical Centre, Albinusdreef 2, 2333 ZA Nijmegen, The Netherlands

5 Department of Urology, Radboud University Medical Centre, Geert Grooteplein-Zuid 10, 6525 GA Nijmegen, The Netherlands $p<0.01)$. The final insufflation pressure was $7.7 \mathrm{mmHg}$ in patients with deep NMB as compared to $9.1 \mathrm{mmHg}$ with moderate NMB $(p=0.19)$. The cumulative opiate consumption during the first $48 \mathrm{~h}$ was significantly lower in patients receiving deep NMB, while postoperative pain scores were similar. In four patients allocated to a moderate $\mathrm{NMB}$, a significant intraoperative complication occurred, and in two of these patients a conversion to an open procedure was required.

Conclusions Our data show that deep NMB facilitates the use of low-pressure PNP during laparoscopic donor nephrectomy by improving the quality of the surgical field. The relatively high incidence of intraoperative complications indicates that the use of low pressure with moderate NMB may compromise safety during LDN. Clinicaltrials.gov identifier: NCT 02602964.

Keywords Deep neuromuscular block - Laparoscopic donor nephrectomy · Laparoscopy · Low-pressure pneumoperitoneum · Pneumoperitoneum

$\begin{array}{ll}\text { Abbreviations } \\ \text { EBL } & \text { Estimated blood loss } \\ \text { HALDN } & \text { Hand-assisted laparoscopic donor nephrectomy } \\ \text { LDN } & \text { Laparoscopic donor nephrectomy } \\ \text { ORT } & \text { Operation time } \\ \text { NMB } & \text { Deep neuromuscular block } \\ \text { PNP } & \text { Pneumoperitoneum } \\ \text { PTC } & \text { Post-tetanic count } \\ \text { QoR40 } & \text { Quality of recovery } \\ \text { TOF } & \text { Train-of-four } \\ \text { WIT1 } & \text { First warm ischemia time }\end{array}$




\section{Introduction}

Laparoscopic donor nephrectomy (LDN), in most countries the "gold standard" for live kidney donation, is associated with an improved quality of life, earlier return to work, and improved cosmetics [1-3]. Modifications of the standard transperitoneal laparoscopic approach, such as the handassisted and/or retroperitoneoscopic approach, have been introduced to refine the surgical technique. However, till date no evidence exists that the hand-assisted and/or retroperitoneoscopic approach improve the clinical outcome after LDN as compared to the standard transperitoneal procedure [4]. Less invasive technique modifications such as laparoendoscopic single site (LESS) or natural orifice transluminal (NOTES) improve the cosmetic result, but are associated with a longer learning curve and higher costs [5-8]. An alternative, simple, and therefore attractive method to refine the standard transperitoneal technique is the use of low-pressure PNP. There is accumulating evidence that low-pressure PNP reduces postoperative pain scores and analgesic consumption [9-12]. Furthermore, low-pressure PNP is better tolerated in cardiac-compromised patients. However, a lower intra-abdominal pressure during laparoscopy comes at a cost. It may compromise surgical conditions, such as working space and sight at the surgical field. A pilot study by our group showed that the use of low pressure during LDN was feasible, but increased duration of surgery [11]. A possible solution to this problem is the application of a deep neuromuscular block (NMB). Deep NMB may provide a better relaxation of the diaphragm and abdominal wall musculature as compared to a moderate NMB during laparoscopy and may thereby increase the space between the abdominal wall and intraabdominal organs during laparoscopy. In a recent study by Kim et al., it was shown that the intra-abdominal pressure could be titrated from $12 \mathrm{mmHg}$ to $9.3 \mathrm{mmHg}$ in patients receiving a deep NMB during laparoscopic colorectal surgery, while intra-abdominal pressure was kept at $12 \mathrm{mmHg}$ in patients allocated to a moderate NMB to maintain adequate surgical conditions [13]. Interestingly, this study showed that the surgical conditions were significantly better in patients receiving a deep NMB despite the use of a lower mean intra-abdominal pressure. However, Staehr-Rye et al. concluded that deep NMB only marginally improved surgical conditions during low-pressure laparoscopic cholecystectomy [14]. Therefore, the question whether deep NMB facilitates the use of lowpressure PNP during laparoscopy remains controversial. To address this issue, we perform a study in which we hypothesize that deep NMB improves surgical conditions during laparoscopic donor nephrectomy with low-pressure PNP.

\section{Methods}

\section{Patients}

This study was performed between April 2015 and February 2016 at the Leiden University Medical Center (Leiden, the Netherlands) and the Radboud University Medical Center (Nijmegen, the Netherlands). All adult patients eligible for LDN were approached at least 2 weeks before surgery. Exclusion criteria included insufficient knowledge of the Dutch language to read the patient information and fill out the questionnaires, chronic use of analgesics or psychotropic drugs, known or suspect allergy to rocuronium or sugammadex. The trial was registered at trials.gov (NCT 02602964).

\section{Ethics}

Ethical approval for this study (Ethical Committee file number: 2014-1322/NL-number: 50874.091.14) was provided by the 'Commissie Mensgebonden Onderzoek Arnhem-Nijmegen', Geert Grooteplein-zuid 10, 6525 GA, Nijmegen, the Netherlands (Chairperson Prof. E. van Leeuwen) on November 10, 2014.

\section{Randomization and blinding}

Patients were randomized using a computer-generated randomization code and assigned to either group 1: lowpressure PNP (6 mmHg) and deep NMB (PTC 1-5) or group 2: low-pressure PNP $(6 \mathrm{mmHg})$ and normal NMB (TOF 0-1). Surgeons, scrub nurses, and the researchers were blinded for allocation of the treatment.

\section{Anesthesia}

Anesthesia was induced by administering $1-3 \mathrm{mg} / \mathrm{kg}$ propofol and $0.2-0.5 \mu \mathrm{g} / \mathrm{kg}$ sufentanil. The TOF-watch (TOF-watch SX, MSD BV, the Netherlands) was calibrated before the administration of NMB agents. First, a tetanic ulnar nerve stimulation was applied, and subsequently the TOF-watch was calibrated. To ensure adequate calibration, 3 TOF measurements were performed; when these 3 measurements differed $>5 \%$, the TOF-watch was recalibrated. For patients in group 1 , rocuronium $1 \mathrm{mg} / \mathrm{kg}$ and for patients in group 2, rocuronium $0.6 \mathrm{mg} / \mathrm{kg}$ was administered. 0.05-0.5 $\mu \mathrm{g} / \mathrm{kg} / \mathrm{h}$ Sufentanil and sevoflurane (1 MAC) were used to maintain anesthesia. For patients in group 1, a continuous infusion of rocuronium was used to maintain deep NMB. The infusion was started at $0.3 \mathrm{mg} /$ $\mathrm{kg} / \mathrm{h}$ but could be adjusted when post-tetanic count (PTC) was 0 or $>5$. PTC was measured every $15 \mathrm{~min}$. In group 2 , 
TOF was measured every $15 \mathrm{~min}$, and in case of TOF $>1$, an extra dose of rocuronium was administered. In all patients, sugammadex $4 \mathrm{mg} / \mathrm{kg}$ was administered after surgery. Patients were extubated when TOF ratio was at least $90 \%$.

\section{Surgical procedure}

At the LUMC, a Veress needle was used to establish a pneumoperitoneum, and at Radboud University Medical Center, a Hasson trocar was introduced under direct vision. In both the hospitals, the camera trocar, two $5 \mathrm{~mm}$ trocars, and one 10/12 mm trocar were subsequently introduced. The hepatic or splenic flexure was mobilized. Gerota's fascia was opened and the renal artery, vein, and ureter were identified. When present, the gonadal, suprarenal, and/or lumbal vein were clipped and transected. Subsequently, the kidney was mobilized and the ureter was transected. A pfannenstiel incision was made, the renal artery and vein were dissected using the endostapler ${ }^{\circledR}$ or vascular clips. After extraction, the kidney was flushed at the back table using cold preservation solution. Afterward, the abdominal cavity was inspected and hemostasis was performed.

\section{Evaluation of perioperative conditions}

After introduction of the camera trocar, after introduction of the third trocar, every 15 min during dissection of the kidney, and at transection of the renal artery, surgical conditions were evaluated using a modified Leiden-Surgical Rating Scale (L-SRS). The L-SRS is a Likert scale ranging from 1 to 5 where 1 indicates extremely poor conditions, 2 poor conditions, 3 acceptable conditions, 4, good conditions, and 5 optimal conditions [15]. The L-SRS aims to quantify the quality of the surgical field based on visibility, surgical space, muscle contractions, handling tactics, and patient movement. In two previous studies, the L-SRS was used in retroperitoneal surgery and consistent L-SRS scorings depending on the depth of NMB were observed without any effect of other factors such as duration of surgery, ventilator settings, and level of arterial $\mathrm{PCO}_{2}[15,16]$. Three separate scores were asked for (1) visibility, (2) working space, and (3) muscle contractions as well as an overall score. When the overall score was below 4 (good conditions), intra-abdominal pressure was increased in steps of $2 \mathrm{mmHg}$. For group 2, in case of insufficient perioperative conditions despite an intra-abdominal pressure of $12 \mathrm{mmHg}$, NMB could be converted to a deeper NMB. When insufficient peroperative surgical conditions persisted despite deep NMB and normal intraabdominal pressure, the surgeon was allowed to handle according to regular protocols, e.g., further increase the intra-abdominal pressure and/or convert to open donor nephrectomy (ODN).

\section{Outcome measures}

The primary outcome measure was the mean peroperative SRS, measured after trocar introduction and every $15 \mathrm{~min}$ thereafter. Secondary outcome measures included operation time (ORT), abdominal pressure, need to increase intra-abdominal pressure, first warm ischemia time (WIT1), estimated blood loss (EBL), perioperative complications, postoperative pain scores, postoperative complications, and postoperative serum creatinine levels. To assess whether the primary surgeon was adequately blinded, he was asked to guess at the end of the procedure whether deep or standard NMB was used.

\section{Sample size calculation and data analysis}

An adapted version of the L-SRS was used for this study. A difference of 0.5 points on the SRS score was used as smallest clinical relevant difference. In the study by Martini et al., mean L-SRS was 4.0 points [15]. For sample size calculations, a standard deviation (SD) of 0.5 was used. A sample size of 17 patients per group was required to obtain a power of $80 \%$ with an alpha of 0.05 . Continuous variables were expressed as mean $\pm \mathrm{SD}$; categorical data as number (percentage). Data were analyzed on an intentionto-treat basis. A Student $t$ test was used to compare normally distributed data. All analyses were performed using SPSS version 22 (IMB Corp. Released 2013. IBM SPSS Statistics for Windows, Version 22.0. Armonk, NY: IBM Corp).

\section{Results}

\section{Patient characteristics}

A total of 34 patients were randomized. Patient demographics are shown in Table 1. There were no significant differences in baseline characteristics. A slight imbalance occurred during block randomization; therefore, 15 patients were randomized to deep NMB and 19 patients to standard NMB.

\section{Per- and postoperative outcomes}

Mean SRS was 4.5 in group 1 (deep NMB) versus 4.0 in group 2 (moderate NMB) $(p=0.01)$, Table 2 . No significant differences were found in the following peroperative parameters: ORT, PNP duration, EBL, and WIT1. For the patients who received moderate NMB, 9 procedures were 
Table 1 Baseline characteristics

Table 2 Peroperative parameters

\begin{tabular}{llll}
\hline & Deep NMB $(n=15)$ & Standard NMB $(n=19)$ & $p$ value \\
\hline Male:female & $9: 6$ & $13: 6$ & 0.61 \\
BMI & $24.7 \pm 3.7$ & $26.2 \pm 3.7$ & 0.22 \\
Right kidney & $5(33 \%)$ & $4(21 \%)$ & 0.42 \\
\hline
\end{tabular}

\begin{tabular}{|c|c|c|c|}
\hline & Deep NMB $(n=15)$ & Standard NMB $(n=19)$ & $p$ value \\
\hline \multicolumn{4}{|c|}{ Mean Surgical Rating Scale (0-5) } \\
\hline SRS first trocar & $4.3 \pm 0.7$ & $3.8 \pm 0.8$ & 0.07 \\
\hline SRS third trocar & $4.3 \pm 0.7$ & $3.9 \pm 0.8$ & 0.13 \\
\hline $\mathrm{SRS}$ at $15 \mathrm{~min}$ & $4.5 \pm 0.5$ & $4.1 \pm 0.6$ & $\mathbf{0 . 0 3}$ \\
\hline SRS at $30 \mathrm{~min}$ & $4.4 \pm 0.5$ & $4.2 \pm 0.5$ & 0.12 \\
\hline SRS at $45 \mathrm{~min}$ & $4.4 \pm 0.5$ & $4.2 \pm 0.5$ & 0.12 \\
\hline \multicolumn{4}{|l|}{ SRS at $60 \mathrm{~min}$} \\
\hline \multicolumn{4}{|l|}{ SRS at $75 \mathrm{~min}$} \\
\hline Overall SRS ${ }^{1}$ & $4.5 \pm 0.5$ & $4.0 \pm 0.4$ & 0.01 \\
\hline \multicolumn{4}{|c|}{ Mean Intra-abdominal pressure $(\mathrm{mmHg})$} \\
\hline IAP first trocar & 6.0 & 6.0 & 1.0 \\
\hline IAP third trocar & $6.4 \pm 1.1$ & $7.2 \pm 2.0$ & 0.21 \\
\hline IAP at $15 \mathrm{~min}$ & $6.5 \pm 1.2$ & $7.4 \pm 2.1$ & 0.18 \\
\hline IAP at $30 \mathrm{~min}$ & $6.8 \pm 1.5$ & $7.7 \pm 2.3$ & 0.21 \\
\hline IAP at $45 \mathrm{~min}$ & $6.9 \pm 1.8$ & $7.8 \pm 2.5$ & 0.26 \\
\hline \multicolumn{4}{|l|}{ IAP at $60 \mathrm{~min}$} \\
\hline \multicolumn{4}{|l|}{$\mathrm{IAP}$ at $75 \mathrm{~min}$} \\
\hline Primary surgeon $<50$ LDNs & 6 & 11 & 0.49 \\
\hline Operation duration (min) & $143 \pm 34.7$ & $159 \pm 45.4$ & 0.26 \\
\hline PNP duration (min) & $136 \pm 63.9$ & $138 \pm 47.0$ & 0.89 \\
\hline EBL (ml) & $137 \pm 199$ & $331 \pm 603$ & 0.21 \\
\hline First WIT (s) & $290 \pm 118$ & $372 \pm 293$ & 0.30 \\
\hline
\end{tabular}

$p$ values $<0.05$ are given in bold

${ }^{\mathrm{a}}$ Primary endpoint

completed with low PNP at $6 \mathrm{mmHg}$, in ten procedures it was necessary to increase the pressure to $8 \mathrm{mmHg}$ or higher (Fig. 1). Eight of 15 procedures with deep NMB were completed with low PNP at $6 \mathrm{mmHg}$. Mean abdominal pressure at the transection of the artery (final stage of the procedure) was $7.7 \mathrm{mmHg}$ in the deep NMB group versus $9.1 \mathrm{mmHg}$ in the standard NMB group $(p=0.21)$. Pain scores were comparable in both groups, see Table 3 . However, opiate use on day 1 and cumulative opiate use after $48 \mathrm{~h}$ were significantly lower in the deep NMB group $(p=0.05)$.

\section{Safety}

Conversion to ODN was necessary in two patients to control an arterial bleeding, Table 4. Both patients had been allocated to normal NMB. In the first patient, the pressure had been increased to $8 \mathrm{mmHg}$ due to insufficient surgical conditions. Conversion to ODN was required due to iatrogenic injury of the renal upper pole artery. In the second patient, conversion was required after an iatrogenic injury of the renal artery. At this point, the intra-abdominal pressure was already increased to normal $(12 \mathrm{mmHg})$ due to insufficient visibility. In both the cases, further postoperative recovery was uncomplicated. In two other patients allocated to a moderate NMB, other intraoperative complications occurred after conversion of the IAP to $12 \mathrm{mmHg}$ for insufficient conditions. In one patient, a bleeding of a lumbal vein occurred and in another patient, the proximal ureter was pulled into the endostapler ${ }^{\circledR}$ and transected during extraction of the kidney.

\section{Assessment of blinding}

In $60 \%$ of the patients operated with deep NMB, the surgeon guessed that deep NMB was used. And when normal NMB was used, in $42 \%$ cases the correct depth of NMB was guessed. 
Fig. 1 Study flow chart

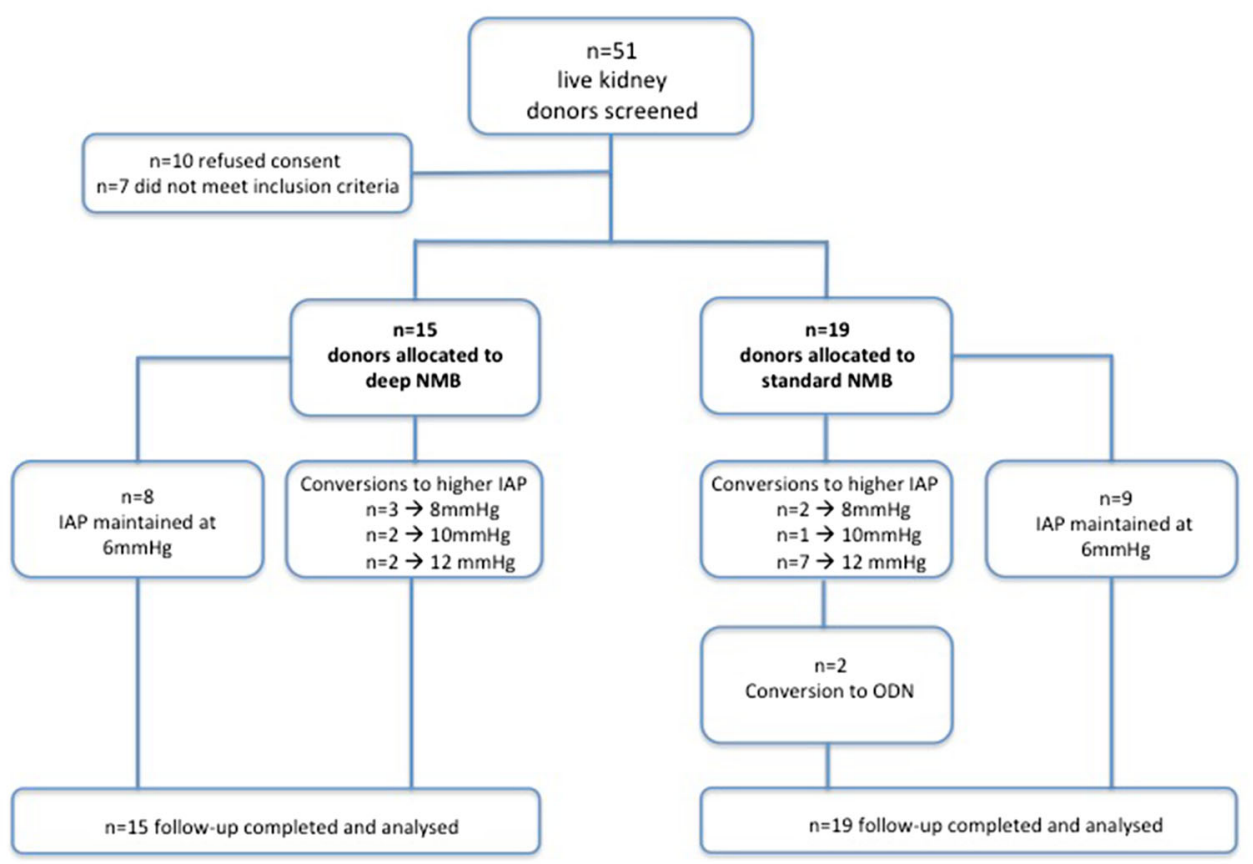

Table 3 Postoperative pain and analgesics

Deep NMB $(n=15) \quad$ Standard NMB $(n=19) \quad p$ value

Overall maximum pain score

Postoperative $1 \mathrm{~h}$

Postoperative day 1

Superficial wound component

Postoperative $1 \mathrm{~h}$

Postoperative $1 \mathrm{~h}$ (movement)

Postoperative day 1

Postoperative day 1 (movement)

Deep intra-abdominal component

Postoperative $1 \mathrm{~h}$

Postoperative $1 \mathrm{~h}$ (movement)

Postoperative day 1

Postoperative day 1 (movement)

Referred shoulder component

\section{Postoperative $1 \mathrm{~h}$}

Postoperative $1 \mathrm{~h}$ (movement)

Postoperative day 1

Postoperative day 1 (movement)

Analgesic medication

Opiate use day $0(\mathrm{mg})$

Opiate use day $1(\mathrm{mg})$

Opiate use day $2(\mathrm{mg})$

Cumulative opiate use $48 \mathrm{~h}$ (mg)

\section{$4.5 \pm 2.5$}

$4.5 \pm 2.6$

$1.6 \pm 1.4$

$2.3 \pm 1.6$

$1.0 \pm 1.3$

$2.3 \pm 1.9$

$1.5 \pm 1.7$

$2.1 \pm 1.9$

$1.2 \pm 1.4$

$1.9 \pm 2.0$

$0.0 \pm 0.0$

$0.1 \pm 0.5$

$1.7 \pm 2.5$

$2.0 \pm 2.7$

17

4

1

22
$4.8 \pm 2.6$

$4.4 \pm 2.4$

$1.2 \pm 1.3$

$2.8 \pm 2.2$

$1.9 \pm 1.8$

$3.1 \pm 2.4$

$1.1 \pm 1.0$

$1.8 \pm 1.4$

$1.3 \pm 1.4$

$2.3 \pm 1.8$

$0.1 \pm 0.3$

$0.2 \pm 0.4$

$1.9 \pm 2.2$

$2.1 \pm 2.7$

19

9

3

31
0.74

0.91

0.48

0.44

0.13

0.27

0.53

0.61

0.79

0.60

0.20

0.87

0.80

0.89

$p$ values $<0.05$ are given in bold
0.55

0.04

0.19

0.05 
Table 4 Conversions, intra-, and postoperative complications

\begin{tabular}{llll}
\hline & Deep NMB $(n=15)$ & Standard NMB $(n=19)$ & $p$ value \\
\hline Conversions & 7 & 10 & 1.0 \\
Conversions to higher IAP & 3 & 2 & \\
$8 \mathrm{mmHg}$ & 2 & 1 & 0.20 \\
$10 \mathrm{mmHg}$ & 2 & 7 & 0.11 \\
$12 \mathrm{mmHg}$ & 0 & 2 & \\
Conversion to ODN & 0 & 4 & \\
Intraoperative complications & 0 & 2 & \\
Arterial bleeding & 0.61 \\
Venous bleeding & 0 & 1 & \\
Ureter transection & 1 & 1 & \\
Postoperative complications & 0 & 3 & \\
Hematoma Pfannenstiehl & 0 & 1 & \\
Vasovagal collaps & 0 & 1 & \\
Transient neuralgia & 1 & 0 & \\
Hypertension & 0 & 1 & \\
\hline
\end{tabular}

${ }^{\text {a }}$ In both cases conversion to ODN

\section{Discussion}

This study shows that a deep NMB facilitates the use of low-pressure PNP during LDN by improving the quality of the surgical field. The surgical conditions as quantified by the Leiden-SRS were significantly better in patients allocated to a deep NMB. Similar results were found in another recent study by Kim et al. [14]. In the study by Kim et al., deep NMB was used in patients undergoing laparoscopic colorectal surgery with titration of the intra-abdominal pressure of $12 \mathrm{mmHg}$ in patients with a moderate NMB, meaning that the insufflation pressure was kept at $12 \mathrm{mmHg}$ in all patients allocated to a moderate NMB. In our study, the insufflation pressure was titrated from $6 \mathrm{mmHg}$ until good or optimal surgical conditions were reached. In nine patients of 19 patients allocated to a moderate $\mathrm{NMB}$, the procedure was completed with a pressure of $6 \mathrm{mmHg}$. This suggests that the titration of the insufflation pressure from low to high results in more procedures completed with a low insufflation pressure. However, in our study, we observed four intraoperative complications in patients allocated to a moderate NMB and in two cases a conversion to ODN was required due to severe bleeding. This strongly indicates that the safety of a low insufflation pressure with a moderate NMB is hampered.

A recent study by Madsen et al. showed that the incidence of referred shoulder pain was lower in women allocated to a low insufflation pressure and deep NMB $(8 \mathrm{mmHg})$ as compared to women allocated to a standard insufflation pressure $(12 \mathrm{mmHg})$ with a moderate NMB [17]. This is in line with a recent systematic review and meta-analysis revealing that the use of low-pressure PNP during laparoscopy is associated with reduced overall and referred shoulder pain scores after laparoscopy [10]. This study showed that patients allocated to a deep NMB consumed significantly less opiates during the first $48 \mathrm{~h}$ after surgery with similar pain scores. This reduction in opiate consumption could at least partly be attributed to the lower mean intra-abdominal pressure in patients allocated to a deep NMB (7.7 versus $9.1 \mathrm{mmHg}$ ). However, an alternative explanation might be that the use of a deep NMB directly affects postoperative pain scores [18]. In theory, a deep NMB allows an increased stretching of the abdominal wall muscle fibers during the insufflation of carbon dioxide [19]. Subsequently this may reduce stretch-induced abdominal pain after laparoscopy.

The main strengths of this study are related to its design as a randomized controlled study in which the blinding of the surgeons was accurate. Also a relatively homogenous population was studied, as live kidney donors are relatively young and in good health, thereby reducing the risk of confounding bias. An important limitation of our study is that the sample size is relatively small. Although the study is adequately powered regarding the primary endpoint, results regarding the secondary endpoints should be interpreted with care.

\section{Conclusion}

In conclusion, our data show that deep NMB facilitates the use of low-pressure PNP during laparoscopic donor nephrectomy by improving the quality of the surgical field. 
Given the relatively high incidence of intraoperative complications and conversions to ODN, the safety of lowpressure PNP with moderate NMB may be hampered.

Acknowledgements We thank Mrs. Kallenberg-Lantrua, Mrs. Meershoek-Klein Kranenbarg, and Mrs. Vote-van den Brink for their assistance.

\section{Compliance with ethical standards}

Disclosures Prof.dr. A. Dahan and dr. M.C. Warlé received grants from Merck Sharp and Dohme for investigator-driven studies unrelated to this study. Mrs. D. Özdemir- van Brunschot, dr. A.E. Braat, Mr. M.F. van der Jagt, prof.dr. G.J. Scheffer, dr. C.H. Martini, dr. J.F. Langenhuijsen, Mrs. R.E. Dam, dr. V.A. Huurman, dr. D. Lam, and dr. F.C. d'Ancona have no conflicts of interest or financial ties to disclose.

Open Access This article is distributed under the terms of the Creative Commons Attribution 4.0 International License (http://crea tivecommons.org/licenses/by/4.0/), which permits unrestricted use, distribution, and reproduction in any medium, provided you give appropriate credit to the original author(s) and the source, provide a link to the Creative Commons license, and indicate if changes were made.

\section{References}

1. Wilson CH, Bhatti AA, Rix DA, Soomro NA (2005) Comparison of laparoscopic and open donor nephrectomy: UK experience. BJU Int 95(1):131-135

2. Kok NF, Lind MY, Hansson BM, Pilzecker D, Mertens zur Borg IR, Knipscheer BC et al (2006) Comparison of laparoscopic and mini incision open donor nephrectomy: single blind, randomised controlled clinical trial. BMJ 333(7561):221

3. Fonouni H, Mehrabi A, Golriz M, Zeier M, Muller-Stich BP, Schemmer P et al (2014) Comparison of the laparoscopic versus open live donor nephrectomy: an overview of surgical complications and outcome. Langenbeck's Arch Surg 399(5):543-551

4. Ozdemir-van Brunschot DM, Koning GG, van Laarhoven KC, Ergun M, van Horne SB, Rovers MM et al (2015) A comparison of technique modifications in laparoscopic donor nephrectomy: a systematic review and meta-analysis. PloS ONE 10(3):e0121131

5. Freeman L, Rahmani EY, Burgess RC, Al-Haddad M, Selzer DJ, Sherman $S$ et al (2011) Evaluation of the learning curve for natural orifice transluminal endoscopic surgery: bilateral ovariectomy in dogs. Vet Surg 40(2):140-150 Epub 2011/01/13

6. Autorino R, Cadeddu JA, Desai MM, Gettman M, Gill IS, Kavoussi LR et al (2011) Laparoendoscopic single-site and natural orifice transluminal endoscopic surgery in urology: a critical analysis of the literature. Eur Urol 59(1):26-45 Epub 2010/09/11

7. Carus T (2013) Current advances in single-port laparoscopic surgery. Langenbeck's Arch Surg 398(7):925-929 Epub 2013/09/17
8. Borle FR, Mehra B, Ranjan Singh A (2015) Comparison of cosmetic outcome between single-incision laparoscopic cholecystectomy and conventional laparoscopic cholecystectomy in rural indian population: a randomized clinical trial. Indian J Surg 77(Suppl 3):877-880 Epub 2016/03/25

9. Gurusamy KS, Samraj K, Davidson BR (2009) Low-pressure versus standard pressure pneumoperitoneum in laparoscopic cholecystectomy. Cochrane Database Syst Rev. doi:10.1002/ 14651858.CD006930.pub2

10. Ozdemir-van Brunschot DM, van Laarhoven KC, Scheffer GJ, Pouwels S, Wever KE, Warle MC (2015) What is the evidence for the use of low-pressure pneumoperitoneum? A systematic review. Surg Endosc. 30(5):2049-2065

11. Warle MC, Berkers AW, Langenhuijsen JF, van der Jagt MF, Dooper PM, Kloke HJ et al (2013) Low-pressure pneumoperitoneum during laparoscopic donor nephrectomy to optimize live donors' comfort. Clin Transplant 27(4):E478-E483 Epub 2013/06/26

12. Singla S, Mittal G, Raghav Mittal RK (2014) Pain management after laparoscopic cholecystectomy-a randomized prospective trial of low-pressure and standard pressure pneumoperitoneum. J Clin Diagn Res 8(2):92-94

13. Kim MH, Lee KY, Lee KY, Min BS, Yoo YC (2016) Maintaining optimal surgical conditions with low insufflation pressures is possible with deep neuromuscular blockade during laparoscopic colorectal surgery: a prospective, randomized, double-blind, parallel-group clinical trial. Medicine 95(9):e2920

14. Staehr-Rye AK, Rasmussen LS, Rosenberg J, Juul P, Lindekaer AL, Riber C et al (2014) Surgical space conditions during lowpressure laparoscopic cholecystectomy with deep versus moderate neuromuscular blockade: a randomized clinical study. Anesth Analg 119(5):1084-1092 Epub 2014/07/01

15. Martini CH, Boon M, Bevers RF, Aarts LP, Dahan A (2014) Evaluation of surgical conditions during laparoscopic surgery in patients with moderate vs deep neuromuscular block. Br J Anaesth 112(3):498-505 Epub 2013/11/19

16. Boon M, Martini C, Hellinga M, Bevers R, Aarts L, Dahan A (2016) Influence of variations in arterial $P \mathrm{CO}_{2}$ on surgical conditions during laparoscopic retroperitoneal surgery. $\mathrm{Br} \mathrm{J}$ Anaesth 117(1):59-65. doi:10.1093/bja/aew114

17. Madsen MV, Istre O, Staehr-Rye AK, Springborg HH, Rosenberg J, Lund J et al (2016) Postoperative shoulder pain after laparoscopic hysterectomy with deep neuromuscular blockade and lowpressure pneumoperitoneum: a randomised controlled trial. Eur J Anaesthesiol 33(5):341-347 Epub 2015/10/20

18. Bruintjes MH, van Helden EV, Braat AE, Dahan A, Scheffer GJ, van Laarhoven CJ et al (2017) Deep neuromuscular block to optimize surgical space conditions during laparoscopic surgery: a systematic review and meta-analysis. Br J Anaesth 118(6):834842. doi:10.1093/bja/aex116

19. van den Bussche EDB, Feryn T, Mulier JP (2007) Effect of muscle relaxants on the abdominal pressure volume relation in bariatric laparoscopic surgery. Surg Endosc 21:S215 